\title{
Prevalence of Onychomycosis among Patients with Transverse Overcurvature of the Nail: Results of a Cross-Sectional Study
}

\author{
Leandro Fonseca Noriega ${ }^{a} \quad$ Nilton Gioia Di Chiacchio ${ }^{a, b}$ \\ Cristina Diniz Borges Figueira de Mello ${ }^{c}$ Maria Victoria Suarez ${ }^{a}$ \\ Diego Leonardo Bet $^{\mathrm{a}}$ Nilton Di Chiacchio ${ }^{\mathrm{a}}$ \\ aDermatology Clinic, Hospital do Servidor Público Municipal de São Paulo, São Paulo, Brazil; bermatology \\ Department, Faculdade de Medicina do ABC, Santo André, São Paulo, Brazil; 'Dermatology Department, \\ Universidade Estadual de Campinas, São Paulo, Brazil
}

\section{Keywords}

Nails $\cdot$ Nail diseases $\cdot$ Onychomycosis

\begin{abstract}
Introduction: Transverse overcurvature of the nail (TON) represents one of the main reasons for dermatological consultations due to nail conditions. Association between TON and subungual hyperkeratosis is common and simulates onychomycosis, constituting a difficult differential diagnosis by clinical features. Many cases are treated in daily practice with antifungal therapy even without mycological confirmation. The aim of this study was to determine the prevalence of onychomycosis among patients with TON associated with subungual hyperkeratosis. Methods: All subjects with clinical diagnosis of TON and subungual hyperkeratosis were subjected to a thorough clinical history, detailed clinical examination, digital photography, and collection of nail clipping and subungual debris for histopathological and microbiological examination (direct microscopy). Results: Sixtyfive samples were collected from 62 female (95.4\%) and 3 male (4.6\%) participants. Age range was between 34 and 83 years (mean age, 61.2 years). Twenty-eight samples were positive for onychomycosis, giving a $43.07 \%$ prevalence rate of onychomycosis among TON associated with hyperkerato-
\end{abstract}

sis. Discussion/Conclusion: According to our data, the prevalence of onychomycosis in patients with TON associated with subungual hyperkeratosis was $43.07 \%$, confirming the need to rule out fungal infection in TON associated with subungual hyperkeratosis prior to considering any unnecessary antifungal treatment.

(c) 2020 S. Karger AG, Basel

\section{Introduction}

Transverse overcurvature of the nail (TON), also known as "incurvated nail" or "pincer nail deformity," is clinically characterized by an increased transverse curvature along the longitudinal axis of the nail plate in the transverse direction. Curvature severity assessment systems are described in attempt to quantify TON by measuring the width and height index, in addition to the radius of a circle formed by its curvature [1-5]. Baseline values for "normal" transverse curvature are still not well defined due to the variations that can occur between different digits, gender, and age range [3].

The prevalence in general population is unknown; however it is known that great toe nails are usually the most affected when compared to other toes and fingers.

$\begin{aligned} & \text { karger@karger.com } \\ & \text { www.karger.com/sad }\end{aligned}$
Karger ${ }^{\prime /}$

Leandro Fonseca Noriega

Dermatology Clinic, Hospital do Servidor Público Municipal de São Paulo 60 Castro Alves

São Paulo 01532000 (Brazil)

leandronorieg@gmail.com 
The demand for medical care is due to discomfort or pain, which can be spontaneous or triggered by walking, wearing shoes, or minor trauma. TON is eventually associated with ingrown nail and rarely leads to loss of soft tissue and distal phalanx bone resorption [5]. However, the severity of deformity is not directly related to the intensity of the symptoms $[3,4,6]$. The evolution is chronic and recurrent, generating significant cosmetic discomfort and functional damage [4]. The etiology of TON is unknown. There are various reports including hereditary and acquired; however, the underlying pathogenesis is not clearly understood $[4,7]$.

Subungual hyperkeratosis is a common finding in several nail disorders and should relate to those leading to thickening beneath the preformed nail plate, that is, thickening of the nail bed or hyponychium [3]. The association between TON and subungual hyperkeratosis is common and simulates onychomycosis, constituting a difficult differential diagnosis by clinical features.

It is common in daily practice to see patients with TON who have been treated with antifungal therapy without mycological confirmation. Prior confirmation of fungal infection is essential, thus avoiding risks of serious adverse effects of systemic medications, prolonged treatments, and unnecessary costs [8-11]. The aim of this study was to determine the prevalence of onychomycosis among patients with TON associated with subungual hyperkeratosis.

\section{Materials and Methods}

This was an observational cross-sectional epidemiological study. Sixty-five patients attending the outpatient Dermatology Clinic of Hospital do Servidor Público Municipal de São Paulo with clinical diagnosis of TON associated with subungual hyperkeratosis of the great toenail were included between January and July 2016.

Patients under 18 years of age, with diagnosis of psoriasis or nail lichen planus, history of trauma or permanent deformity of nail plate, and patients under topical or systemic antifungal medications in the last 90 and 180 days, respectively, were excluded. All patients were subjected to a thorough clinical history, detailed clinical examination, digital nail photography, and collection of nail clipping and subungual debris for histopathological and microbiological examination (direct microscopy).

For direct microscopy, the sample was placed on a glass slide, clarified with $10 \%$ potassium hydroxide $(\mathrm{KOH})$ with $40 \%$ dimethyl sulfoxide (DMSO), covered by a coverslip, and evaluated by optical microscopy in $\times 10$ and $\times 40$ magnifications. Nail clipping was obtained by cutting a 4-mm fragment from the free edge of the affected nail, with nail nipper introduced in the distal to proximal direction, resulting in a triangular fragment (base formed by the free edge), as shown in Figure 1. This sample was submitted to his-
Table 1. Prevalence of onychomycosis among patients with transverse overcurvature of the nail (TON)

\begin{tabular}{|c|c|c|c|}
\hline & $\begin{array}{l}\mathrm{KOH}+\mathrm{DMSO}^{*} \\
(+)\end{array}$ & $\begin{array}{l}\mathrm{KOH}+\mathrm{DMSO}^{*} \\
(-)\end{array}$ & Total \\
\hline \multicolumn{4}{|c|}{ TON plus onychomycosis } \\
\hline $\mathrm{PAS}^{* *}(+)$ & 4 & 23 & $28(56.93 \%)$ \\
\hline $\mathrm{PAS}^{* *}(-)$ & 1 & & \\
\hline \multicolumn{4}{|l|}{ TON } \\
\hline $\operatorname{PAS}^{* *}(-)$ & & 37 & $37(43.07 \%)$ \\
\hline Total & & & $65(100 \%)$ \\
\hline
\end{tabular}

topathological examination with periodic acid-Schiff (PAS) staining.

Diagnosis of onychomycosis was confirmed by the presence of septate hyphae in direct microscopy and/or in histopathological evaluation of PAS-stained nail clipping (shown in Fig. 2). If only yeast forms were seen on direct microscopy, it was considered negative.

\section{Results}

A total of 65 samples were obtained, of which 62 (95.4\%) were female and $3(4.6 \%)$ were male. Their ages ranged from 34 to 83 (mean age, 61.2) years.

Out of the 65 samples, 28 were considered positive for onychomycosis and 37 were negative, resulting in a prevalence of 43.07 and $56.93 \%$, respectively. One sample was identified positive by direct microscopy with $\mathrm{KOH} /$ DMSO, 23 by PAS staining, and 4 samples by both tests (Table 1).

\section{Discussion/Conclusion}

The etiology of TON is unknown. There are various reports including hereditary and acquired etiologies, the latter associated with specific pathologies of the nail apparatus, systemic diseases, or use of medications [3, 12, 13]. However, the underlying pathogenesis is not clearly understood $[4,7]$.

The association between TON and onychomycosis is also described, but it is not clear whether onychomycosis is a cause or a consequence of TON. Nail plate dystrophy due to fungal infection can alter its curvature, leading to TON. On the other hand, thickening of the nail plate, sub- 


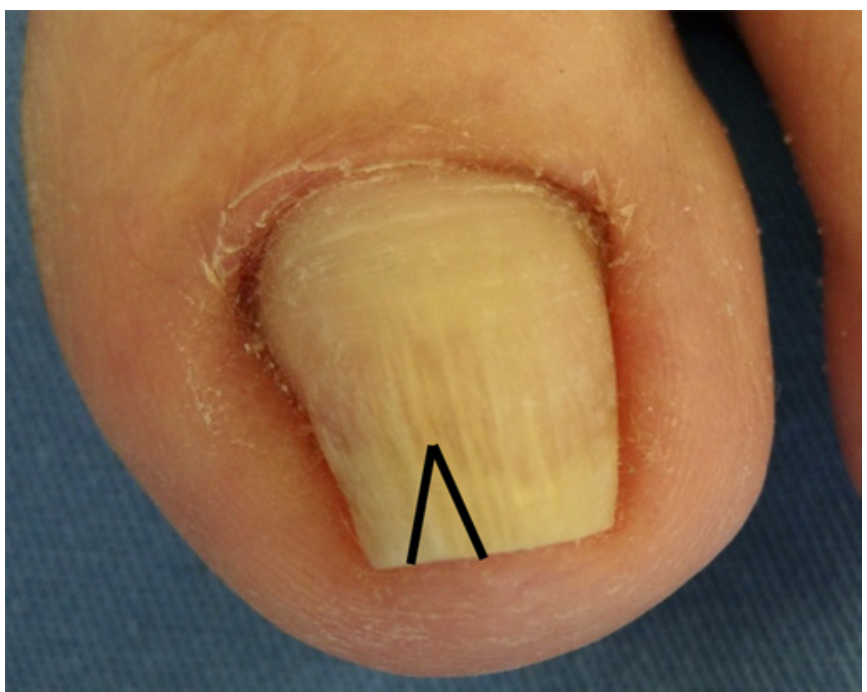

Fig. 1. Nail clipping.

ungual hyperkeratosis, and onycholysis are common findings in TON that increases the susceptibility to fungal infection (onychomycosis) $[4,14]$.

In this study, the age of the patients ranged from 34 to 83 years (mean age 56 and 63 years in the group with and without onychomycosis, respectively). In contrast, Lee et al. [4] found a greater age range in their study (from 10 to 77 years).

Our study revealed a high prevalence of TON in females (95.4\%), when compared to males (4.6\%). Higashi [14] and Lee et al. [4] also reported a higher prevalence of this deformity in females (66.7 and 65.7\%). This difference is probably due to the greater aesthetic concern and greater demand for medical care by women when compared to men.

In 1990, Higashi [14], reported on 6 patients with TON due to onychomycosis (confirmed by direct microscopy and cultures), ages ranging from 56 to 71 years. Deformity resolved in all cases after 6 months of treatment with systemic griseofulvin. According to this author, fungal infection is a cause of TON and the underlying pathogenesis would be the hyperkeratosis of the lateral nail edges, leading to the narrowing and elevation of the distal nail bed. Consequently, there would be a predominance of mechanical forces that induce an increase in transverse nail curvature [14].

Recently, Lee et al. [4] characterized the clinical features of TON in 35 Korean patients and compared conservative and surgical treatment. Onychomycosis was present in $37.1 \%$ of patients. Interestingly, they observed that in the presence of onychomycosis, oral antifungal
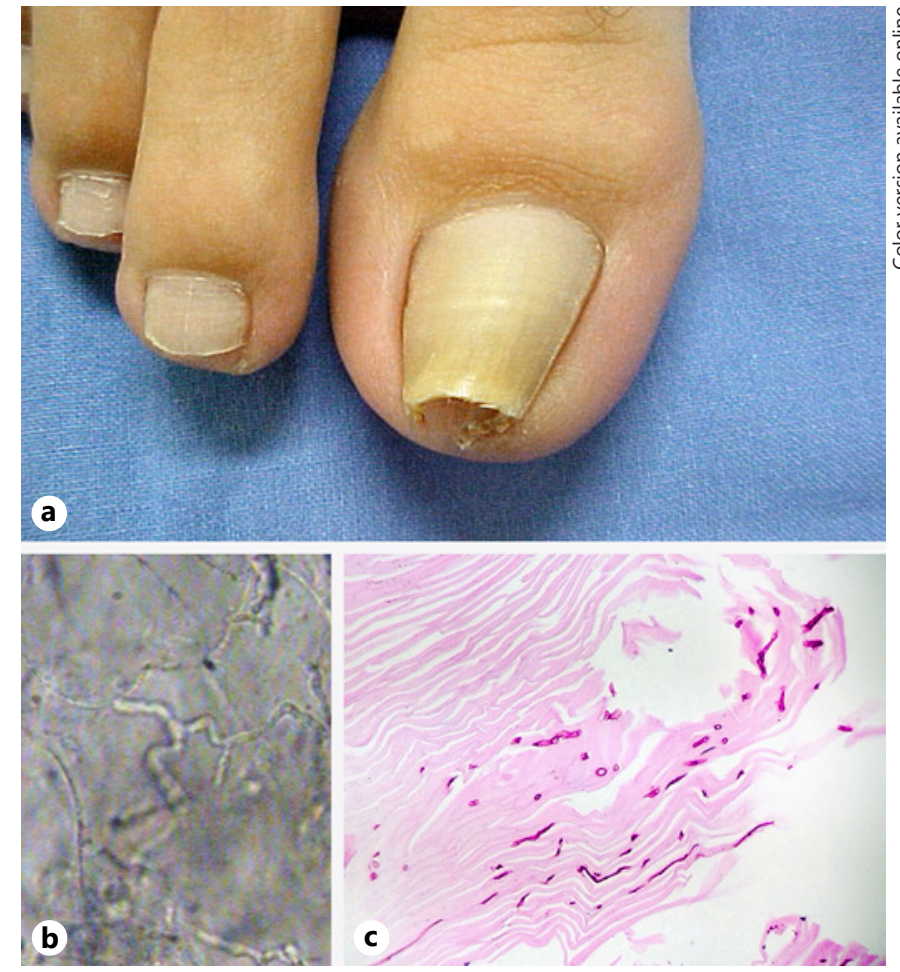

Fig. 2. a Transverse overcurvature of the nail and onychomycosis. b Direct mycological examination: septate hyaline hyphae. c Histopathological evaluation of periodic acid-Schiff (PAS)-stained nail clipping: presence of septate hyphae.

therapy (itraconazole) added to conservative treatment resulted in a more rapid response compared to patients without onychomycosis. The authors suggest that this nail deformity causes the nail plate to be more susceptible to fungal infection and also cite the hypothesis described by Higashi but indicate the need for further studies [4].

In this study, the prevalence of onychomycosis among patients with TON was $43.07 \%$, demonstrating an important relationship between the 2 pathologies. However, since no follow-up was performed, and due to study design, it was not possible to define whether TON was caused primarily by onychomycosis or whether this nail deformity predisposed to fungal infection and this should be explored in future trials.

Analyzing the samples inversely, $56.93 \%$ of patients with TON had no fungal infection. Some of these cases are commonly misdiagnosed as onychomycosis by general dermatologists, or even general physicians, leading to poor responses to therapy and exposing patients to prolonged treatments, risks of serious adverse effects, and unnecessary costs. 
The difficulty of clinical diagnosis of several nail disorders, including the association TON/onychomycosis, is relevant, since many signs and symptoms are similar or often identical. Thus, further investigation is necessary to confirm the diagnosis and consequently guide the specific treatment.

This study demonstrated that the prevalence of onychomycosis in patients with TON associated with subungual hyperkeratosis seems to be increased (43.07\%). This finding may be a general advice to rule out fungal infection in TON associated with subungual hyperkeratosis before treatment.

\section{Acknowledgement}

This work was supported by Fundo de Apoio à Dermatologia do Estado de São Paulo, Sebastião Sampaio - FUNADERSP.

\section{Statement of Ethics}

This project was conducted in accordance with the requirements of Brazilian legislation, the World Medical Association Declaration of Helsinki and in compliance with Good Clinical Prac- tices, starting after approval by the responsible Research Ethics Committee (CAAE: 52005215.8.0000.5442). Patients have given their written informed consent.

\section{Conflicts of Interest Statement}

The authors have no conflicts of interest to declare.

\section{Funding Sources}

The Fundo de Apoio à Dermatologia do Estado de São Paulo, Sebastião Sampaio - FUNADERSP provided financial support for the purchase of materials used in mycological exams.

\section{Author Contributions}

L.F.N. and N.D.C. designed the work, carried out data collection and analysis, wrote the manuscript, performed critical revision of the article, and provided final approval of the version to be published. N.G.D.C. and C.D.B.F.M. wrote the manuscript, performed critical revision of the article, and provided final approval of the version to be published. M.V.S. designed the work, performed critical revision of the article, and provided final approval of the version to be published. D.L.B. performed critical revision of the article and provided final approval of the version to be published.

\section{References}

1 Murdan S. Transverse fingernail curvature in adults: a quantitative evaluation and the influence of gender, age, and hand size and dominance. Int J Cosmet Sci. 2011 Dec;33(6):50913.

2 Cornelius CE, Shelley WB. Pincer nail syndrome. Arch Surg. 1968;96(2):321-2.

3 Baran R, Dawber RPR, Haneke E, Tosti A, Bristow I. A text atlas of nail disorders: techniques in investigation and diagnosis. 3rd ed. London: Taylor \& Francis; 2003.

4 Lee JI, Lee YB, Oh ST, Park HJ, Cho BK. A clinical study of 35 cases of pincer nails. Ann Dermatol. 2011 Nov;23(4):417-23.

5 Kosaka M, Kusuhara H, Mochizuki Y, Mori H, Isogai N. Morphologic study of normal, ingrown, and pincer nails. Dermatol Surg. 2010; 36(1):31-8.
6 Jung DJ, Kim JH, Lee HY, Kim DC, Lee SI, Kim TY. Anatomical characteristics and surgical treatments of pincer nail deformity. Arch Plast Surg. 2015 Mar;42(2):207-13.

7 Twigg EV, Weitz NA, Scher RK, Grossman ME. Pincer nails in a patient with systemic lupus erythematosus and lupus nephritis: a case report. JAAD Case Rep. 2016 Jun;2(3):233-5.

8 Cabete J, Galhardas C, Apetato M, Lestre S. Onychomycosis in patients with chronic leg ulcer and toenail abnormalities. An Bras Dermatol. 2015;90(1):136-9.

9 Almeida LMMd, Souza EAd. F, Bianchin DB, Svidzinski TIE. Resposta in vitro de fungos agentes de micoses cutâneas frente aos antifúngicos sistêmicos mais utilizados na dermatologia. An Bras Dermatol. 2009;84(3):24955.
10 Stephen S, Tosti A, Rubin AI. Diagnostic applications of nail clippings. Dermatol Clin. 2015 Apr;33(2):289-301.

11 Velasquez-Agudelo V, Cardona-Arias JA. Meta-analysis of the utility of culture, biopsy, and direct $\mathrm{KOH}$ examination for the diagnosis of onychomycosis. BMC Infect Dis. 2017; 17(1): 166 .

12 Tully AS, Trayes KP, Studdiford JS. Evaluation of nail abnormalities. Am Fam Physician. 2012 Apr;85(8):779-87.

13 Greiner D, Schöfer H, Milbradt R. Reversible transverse overcurvature of the nails (pincer nails) after treatment with a beta-blocker. J Am Acad Dermatol. 1998 Sep;39(3):486-7.

14 Higashi N. Pincer nail due to tinea unguium. Hifu. 1990;32:40-4. 